BULL. AUSTRAL. MATH. SOC.

\title{
PRESENTATIONS FOR SOME DIRECT PRODUCTS OF GROUPS
}

\author{
P.E. KENNE
}

We give efficient presentations for the direct product of two copies of the alternating group of degree five and the direct product of the alternating group of degree five and the binary icosahedral group.

A finite group $G$ is said to be efficient if it has a presentation on $d$ generators and $d+\operatorname{rank}(M(G))$ relations $(M(G)$ is the Schur multiplicator of $G$, see [1]). It is well known that $A_{5}$, the alternating group of degree five, is efficient because

(i) $M\left(A_{5}\right)$ has order 2 and

(ii) $A_{5}$ has a presentation $\left\langle a, b \mid a^{5}=b^{3}=(a b)^{2}=1\right\rangle$.

Several authors $([1$, p. 191], [4], [6, p. 149]) have recently asked whether the direct product $A_{5} \times A_{5}$ of two copies of $A_{5}$ is efficient. The answer is yes. We show this by first giving an efficient presentation for $A_{5} \times B$, where $B$ is the binary icosahedral group. The method for obtaining this presentation is outlined in the last paragraph.

Let $H$ be defined by

$$
\left\langle x, y \mid y^{6}=x^{4} y x^{-1} y^{-3} x^{-1} y^{-1}=\left(x y^{2}\right)^{2} x^{-1} y^{-1}\left(x y^{-1}\right)^{2}=1\right\rangle .
$$

It is easy to see that $H$ is perfect. Coset enumeration shows that $H$

Received 25 May 1983. 
has order 7200 . It is known that $B$ has a presentation

$$
\left\langle c, d \mid c^{5}=d^{3}=(c d)^{2}\right\rangle,
$$

and the mapping $x \mapsto c d, y \mapsto d$ of $H$ onto $B$ extends to a homomorphism since the relations of $H$ map to the identity of $B$; thus $B$ is a quotient of $H$. From the tables given in [5], it follows that $H$ is $A_{5} \times B$. Since $M\left(A_{5} \times B\right)$ has order 2 (using [1, Theorem 4.11, p. 108]), we have shown that $A_{5} \times B$ is efficient.

Coset enumeration shows that the group obtained by adding the relation $x^{10}=1$ to (*) has order 3600 and is therefore $A_{5} \times A_{5}$. The rank of $M\left(A_{5} \times A_{5}\right)$ is two, and so $A_{5} \times A_{5}$ is also efficient.

These presentations do not shed any light on the problem of whether $B \times B$ is efficient; note that Wiegold [6, p. 149] has conjectured that $B \times B$ is not efficient.

It is clear that $A_{5} \times B$ has a presentation

$$
\begin{aligned}
\langle a, b, c, d| a^{5}=b^{3}=(a b)^{2}=1, c^{5}=d^{3}=(c d)^{2}, \\
{[a, c]=[a, d]=[b, c]=[b, d]=1\rangle }
\end{aligned}
$$

and is generated by the elements $x=$ acd and $y=a b d$. Defining relations for $A_{5} \times B$ in terms of $x$ and $y$ were constructed by using a method due to Cannon [2] as implemented in cayley [3]. This resulted in a two-generator four-relation presentation. The coset enumeration above showed the fourth relation, $\left[x^{2}, y^{2}\right]=1$, to be redundant.

ADDED IN PROOF. B.H. Neumann has asked (private communication) whether the group $K$ defined by

$$
\left\langle x, y \mid x^{10}=y^{6}, x^{4} y x^{-1} y^{-3} x^{-1} y^{-1}=\left(x y^{2}\right)^{2} x^{-1} y^{-1}\left(x y^{-1}\right)^{2}=1\right\rangle
$$

is the other perfect group of order 7200 (the direct product of two copies of $B$ with amalgamated central subgroups, denoted by $P_{4}$ in [5]). Coset enumeration once again shows that $K$ has order 7200 . The normal subgroup lattice of $K$ was calculated using Cayley [3], and it was found that $K$ has no normal subgroup of order 60 . This shows that $K$ is $P_{4}$. 


\section{References}

[1] F. Rudolf Beyl and Jürgen Tappe, Group extensions, representations, and the Schur multiplicator (Lecture Notes in Mathematics, 958. Springer-Verlag, Berlin, Heidelberg, New York, 1982).

[2] John J. Cannon, "Construction of defining relations for finite groups", Discrete Math. 5 (1973), 105-129.

[3] John J. Cannon, "Software tools for group theory", The Santa Cruz conference on finite groups, Santa Cruz, 1979, 495-502 (Proc. Symposia Pure Maths., 37. American Mathematical Society, Providence, Rhode Island, 1980).

[4] Edmund F. Robertson, "Efficiency of finite simple groups and their covering groups", Finite groups - coming of age (Proceedings, Concordia, Montreal, 1982. To appear).

[5] Günter Sandlöbes, "Perfect groups of order less than $10^{4}$ ", Comm. Algebra 9 (1981), 477-490.

[6] J. Wiegold, "The Schur multiplier: an elementary approach", Groups, St Andrews, 1981, 137-154 (London Mathematical Society Lecture Note Series, 71. Cambridge University Press, Cambridge, 1982).

Department of Mathematics, Institute of Advanced Studies, Australian National University, GPO Box 4, Canberra, ACT 2601, Australia. 